\title{
Author Correction: U-Net: deep learning for cell counting, detection, and morphometry
}

Thorsten Falk (1), Dominic Mai, Robert Bensch, Özgün Çiçek, Ahmed Abdulkadir, Yassine Marrakchi, Anton Böhm, Jan Deubner, Zoe Jäckel, Katharina Seiwald, Alexander Dovzhenko, Olaf Tietz, Cristina Dal Bosco, Sean Walsh, Deniz Saltukoglu, Tuan Leng Tay D, Marco Prinz, Klaus Palme, Matias Simons, Ilka Diester, Thomas Brox and Olaf Ronneberger (D)

Correction to: Nature Methods https://doi.org/10.1038/s41592-018-0261-2, published online 17 December 2018

In the version of this paper originally published, one of the affiliations for Dominic Mai was incorrect: "Center for Biological Systems Analysis (ZBSA), Albert-Ludwigs-University, Freiburg, Germany" should have been "Life Imaging Center, Center for Biological Systems Analysis, Albert-Ludwigs-University, Freiburg, Germany." This change required some renumbering of subsequent author affiliations. These corrections have been made in the PDF and HTML versions of the article, as well as in any cover sheets for associated Supplementary Information.

Published online: 25 February 2019

https://doi.org/10.1038/s41592-019-0356-4

\section{Author Correction: Stability, affinity, and chromatic variants of the glutamate sensor iGluSnFR}

Jonathan S. Marvin (D, Benjamin Scholl, Daniel E. Wilson, Kaspar Podgorski, Abbas Kazemipour, Johannes Alexander Müller (1D), Susanne Schoch, Francisco José Urra Quiroz, Nelson Rebola, Huan Bao, Justin P. Little, Ariana N. Tkachuk, Edward Cai, Adam W. Hantman, Samuel S.-H. Wang, Victor J. DePiero, Bart G. Borghuis (D, Edwin R. Chapman, Dirk Dietrich, David A. DiGregorio, David Fitzpatrick and Loren L. Looger (1)

Correction to: Nature Methods https://doi.org/10.1038/s41592-018-0171-3, published online 30 October 2018

The version of this paper originally published cited a preprint version of ref. 12 instead of the published version (Proc. Natl. Acad. Sci. USA 115, 5594-5599; 2018), which was available before this Nature Methods paper went to press. The reference information has been updated in the PDF and HTML versions of the article.

Published online: 28 February 2019

https://doi.org/10.1038/s41592-019-0363-5

\section{Author Correction: Methods to study RNA-protein interactions}

Muthukumar Ramanathan, Douglas F. Porter and Paul A. Khavari (D)

Correction to: Nature Methods https://doi.org/10.1038/s41592-019-0330-1, published online 25 February 2019

In the version of this paper originally published, three references were accidentally omitted: Schwartz, J. C. et al. Cell Rep. 5, 918-925 (2013); Tundup, S. et al. FEBS Lett. 580, 1285-1293 (2006); and Itri, F. et al. Biochem. Biophys. Res. Commun. 492, 67-73 (2017). The PDF and HTML versions of the paper now include these as references 58, 59, and 60, respectively, and subsequent references have been renumbered accordingly.

Published online: 8 March 2019

https://doi.org/10.1038/s41592-019-0366-2 